\title{
Fatigue stress ratio effect on fretting-fatigue crack nucleation: comparison between multi-axial and uni-axial predictions
}

\author{
GANDIOLLE Camille ${ }^{1,}$, , FOUVRY Siegfried ${ }^{1, b}$ \\ ${ }^{1}$ LTDS, Ecole central de Lyon, 36 avenue Guy de Collongue, 69130 Ecully, France \\ ${ }^{a}$ camille.gandiolle@ec-lyon.fr, bsiegfried.fouvry@ec-lyon.fr
}

Keywords: Fretting-fatigue, crack nucleation, Crossland, Haigh analysis, stress ratio effect.

\begin{abstract}
This research focuses on high cycle fretting fatigue crack nucleation prediction. A plastic steel on steel cylinder/plane contact was investigated keeping constant the normal force and the maximum fatigue stress but varying the fatigue stress ratio $\left(\mathrm{R}_{\mathrm{F}}=0.6\right.$ to 1$)$. The evolution of the crack length as function of the applied fretting tangential force amplitude at $10^{6}$ cycles allows us to formalize the crack nucleation condition. It shows that the threshold tangential force marking the crack nucleation (i.e. $b_{p_{-} \text {th }}=0 \mu \mathrm{m}$ ) is not affected by the fatigue stress ratio. But an increase of the fatigue stress amplitude sharply increases the crack extension. To model the experiments, a 2D plastic plain strain FEM modeling is performed. As expected, the computed stress field description is mesh-dependent. However we demonstrate that a representative stress description is obtained at the $3^{\text {rd }}$ node (i.e. $2^{\text {nd }}$ node below the surface). By coupling this mesh condition and non-local critical distance approach, reliable prediction of the crack nucleation risk can be achieved either considering a Crossland multi-axial fatigue analysis or using a basic uni-axial Haigh's description.
\end{abstract}

\section{Introduction}

Fretting fatigue is characterized by combined high stress gradients induced by microdisplacements at the interface (fretting) and homogeneous stresses induced by bulk fatigue stressing. It is usually admitted that under partial slip conditions (i.e. when the interface maintains an inner stuck region) the crack nucleation is controlled by the shear stressing whereas the deeper propagation stage is controlled by the fatigue loading [1]. This failure risk is critical for modern industries, thus predicting crack nucleation is essential. Numerous studies have been conducted using fretting fatigue map concepts, where the cracking domains are reported as a function of the tangential fretting load and the fatigue load. A more recent analysis [2] considers a local stress analysis coupling the effect of contact and fatigue stressing. However very little has been done to formalize the effect of the fatigue stress ratio on fretting fatigue nucleation process.

In this study we focus on a simple fatigue approach to predict small cracks nucleation for various fatigue stress ratios by using first the Crossland multi-axial approach, but also by comparing uniaxial Haigh description. Previous results [3] show that a basic uni-axial fatigue approach can be considered. Transposed to severe plastic stress gradient fretting conditions, this strategy is investigated by considering a non-local critical distance approach taking into account the FEM mesh size influence on the prediction.

\section{Materials and fretting fatigue experiments}

The response of a low alloyed steel flat specimen fretted against a steel cylinder of $4.57 \mathrm{~mm}$ of radius was investigated. The contact was tested in plain fretting $\left(\mathrm{PF}\right.$, no fatigue force, $\left.\sigma_{\mathrm{F}}=0\right)$, and fretting fatigue (FF). A constant high static normal force $\mathrm{P}$ was applied so that severe plastic deformations are induced in the flat specimen. FEM indentation simulations indicate a maximum pressure of $\mathrm{p}_{0}=2.5 \sigma_{\mathrm{y}}$ inducing a maximum von Mises stress of $\sigma_{\mathrm{VM}} \approx 1.4 \sigma_{\mathrm{y}}$. Fatigue samples have been tested at a constant maximal fatigue force $\sigma_{\mathrm{Fmax}}$ but varying fatigue stress ratios (i.e. $\mathrm{R}_{\mathrm{F}}=$ $\sigma_{\text {Fmin }} / \sigma_{\text {Fmax }}=0.6$ to 1$)$. The main mechanical properties of the materials are presented in Table 1 . 
Table 1. Mechanical properties of the tested materials

\begin{tabular}{l|c|c} 
Materials & Flat & Cylinder \\
\hline Young's modulus E $[\mathrm{GPa}]$ & 200 & 200 \\
\hline Poisson's coefficient $\nu$ & 0.3 & 0.3 \\
\hline Yield stress $\sigma_{\mathrm{Y} \_(0.2 \%)}[\mathrm{MPa}]$ & $\sigma_{\mathrm{Y}}{ }^{*}$ & $\sigma_{\mathrm{Y}_{-}}{ }^{*}$ \\
\hline Ultimate stress $\sigma_{\mathrm{UTS}}[\mathrm{MPa}]$ & $\sigma_{\mathrm{UTS}}{ }^{*}$ & $\sigma_{\text {UTS_c }}{ }^{*}$ \\
\hline Fatigue limit $\sigma_{\mathrm{D}}[\mathrm{MPa}]$ & $\sigma_{\mathrm{D}}{ }^{*}$ & $\sigma_{\mathrm{D}_{-}}{ }^{*}$ \\
\hline
\end{tabular}

*confidential values

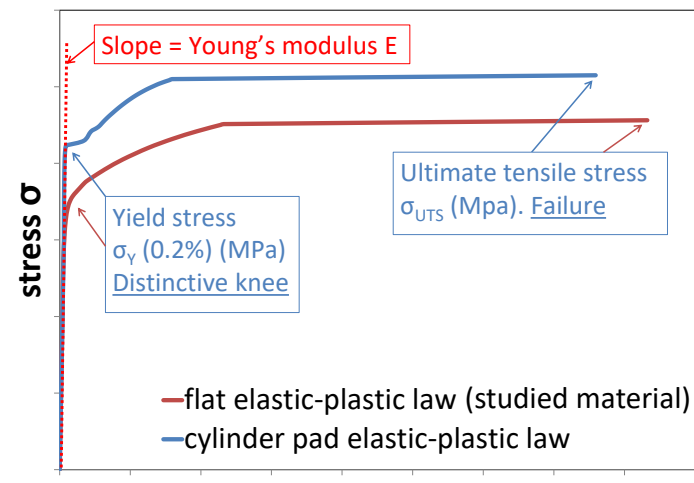

strain $\varepsilon$

Fig. 1. Elastic-plastic law of the tested material

Both materials mechanical responses are described by monotonic elastic-plastic laws determined by simple tensile tests (Fig. 1). Each plain fretting and fretting fatigue test (Fig. 2a) is realized at $12 \mathrm{~Hz}$ for $10^{6}$ cycles. The fretting displacement $\delta(\mathrm{t})$ and fatigue force $\sigma(\mathrm{t})$ are imposed with two independent actuators generating a controlled tangential fretting force $\mathrm{Q}(\mathrm{t})$ in the contact. The crack analysis is restricted to the flat specimen. The dimensions chosen allow plane strain conditions along the central axis of the fretting scar. All the fretting scars were analyzed following the method depicted in Fig. 2b. At the end of the test, the maximum projected crack length is considered and plotted as a function of the tangential force amplitude. The crack nucleation condition can be defined by extrapolating the evolution of $b_{p}$ the projected crack length to the $x$-axis $\left(Q^{*}{ }_{C N}, b_{p} \rightarrow 0\right)$. Using this strategy, various crack nucleation thresholds can be considered depending on the chosen threshold crack length. Hence, assuming a threshold crack length $\mathrm{x}$, defining a crack nucleation condition, it leads to the corresponding $Q^{*}$ threshold tangential loading (Fig. 2c).

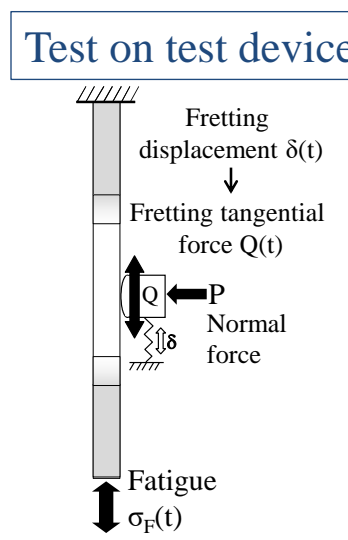

(a)

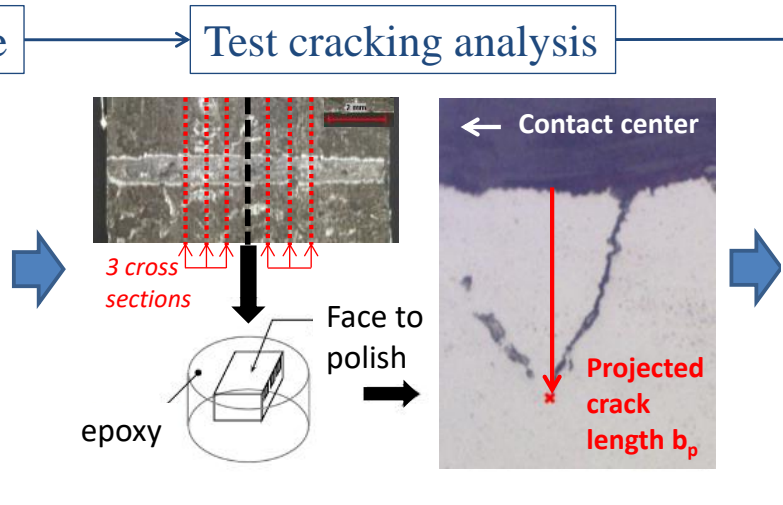

(b)

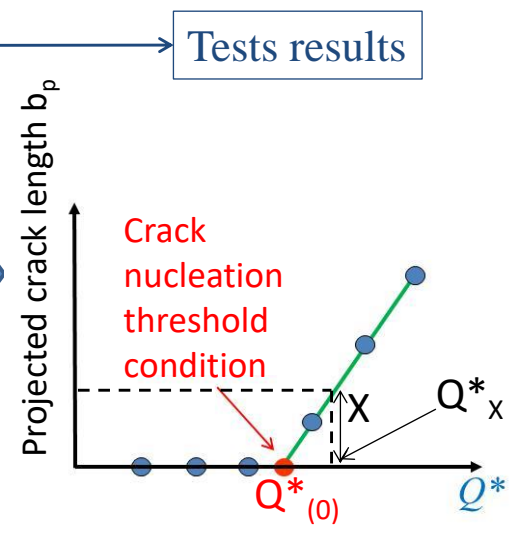

(c)

Fig. 2. (a) Test device, (b) Optical analysis, (c) Crack nucleation threshold determination

\section{Experimental results}

A first set of test was done to establish the evolution of $b_{p}$ as a function of the fretting tangential amplitude $\mathrm{Q}^{*}(\mathrm{~N} / \mathrm{mm})$ for plain fretting conditions. Then fretting fatigue experiments were performed applying a constant maximum fatigue stress $\sigma_{\text {Fmax }}$ but varying the fatigue stress ratio from 0.6 to 1 (i.e. pre-stressed test). For each fatigue condition, the fretting loading was adjusted to establish, like for plain fretting, the evolution of $b_{p}$ as a function of $Q^{*}$. All the analyses were done at $10^{6}$ cycles which provides a high cycle fatigue description of the fretting fatigue process.

Application of a fatigue loading compared to plain fretting does not affect the crack nucleation threshold but increases the crack extension (Fig. 3). Considering the fretting fatigue results, it is interesting to note that for $b_{p_{-} t h}=0$, the crack nucleation is independent of the fatigue stress ratio $R_{F}$ and equal to $\mathrm{Q}^{*} / \mu \mathrm{P}=0.13$. This suggests that the activation of an incipient fretting fatigue crack is 
only controlled by the cyclic contact stressing. For longer cracks the fatigue stress ratio effect is more effective: an increase of the fatigue stress amplitude promotes an extension of the crack propagation. From this analysis the threshold tangential force amplitude related to $b_{p_{-} t h}=0,50$ and $100 \mu \mathrm{m}$ are extrapolated for both plain fretting and fretting fatigue (Table 2).

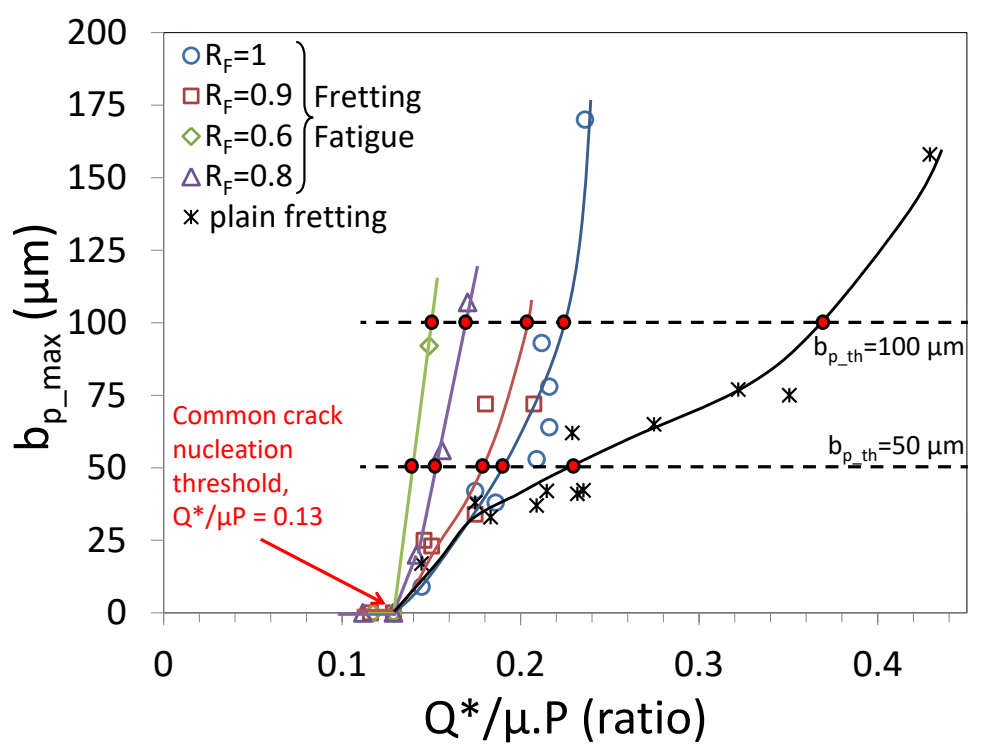

Table 2: Tangential force ratio $\mathrm{Q}^{*} / \mu \mathrm{P}$ related to the crack nucleation threshold conditions $\left(\mathrm{b}_{\mathrm{x}_{-} \mathrm{th}}=0,50\right.$ and $\left.100 \mu \mathrm{m}\right)$

\begin{tabular}{|c|c|c|c|c|c|}
\hline \multirow[t]{2}{*}{$Q * / \mu P$} & \multirow{2}{*}{$P F$} & \multicolumn{4}{|c|}{ Fretting fatigue - $R_{F}$} \\
\hline & & 0.6 & 0.8 & 0.9 & 1 \\
\hline$b_{t h}=0 \mu m$ & 0.13 & 0.13 & 0.13 & 0.13 & 0.13 \\
\hline 50 & 0.23 & 0.14 & 0.15 & 0.18 & 0.19 \\
\hline$b_{t h}=100 \mu \mathrm{m}$ & 0.30 & 0.15 & 0.17 & 0.21 & 0.22 \\
\hline
\end{tabular}

Fig. 3. Fatigue stress ratio effect, experimental plain fretting and fretting fatigue results

\section{Modeling}

Meshing and critical distance considerations. A 2D plain strain finite element model (FEM) has been implemented on Abaqus 6.9. It allows simulation of the multi-axial contact stress state, including the contact plasticity behavior. Fig. 4 a presents a scheme of the model. The Lagrange multiplier with the friction coefficient $\mu=1$ determined before by experimental test was selected as the contact algorithm. The mesh consists of linear elements. Isotropic hardening laws were used both for the cylinder and the flat specimen. The amount of induced plasticity decreases with the number of fretting cycles applied. The number of simulated cycles is adjusted to reach the elastic shakedown condition $(50<\mathrm{N}<100)$. The fatigue post treatment is done on the stabilized stress state. As previously underlined [3], for friction value is higher than 0.3 , the maximum cracking risk is observed on the surface at the contact border $(\mathrm{z}=0, \mathrm{x}=-\mathrm{a}$ with a contact radius) so-called "hot spot".

The contact stressing induces a severe stress gradient [4], and it was shown by Heredia et al. [5] that the stress along the $\mathrm{z}$-axis at the hot spot location is mesh dependent. This tendency is confirmed in Fig. $4 \mathrm{~b}$ where the elastic $\sigma_{11}(\mathrm{x}=-\mathrm{a}, \mathrm{z})$ profiles computed using different mesh sizes are compared versus the exact analytical profile. The analysis shows that FEM computations systematically underestimate the hot spot stress value and the error increases with the mesh size.

Heredia et al. [5] suggest that to perform reliable crack nucleation predictions the fatigue analysis must be done at the $2^{\text {nd }}$ node (i.e. $1^{\text {st }}$ node below the surface). Our analysis shows that the best correlation with the exact analytical stress field is achieved at the $3^{\text {rd }}$ node (i.e. $2^{\text {nd }}$ node below the surface). Indeed whatever the mesh size from $\mathrm{d}=0.1 \mu \mathrm{m}$ to $20 \mu \mathrm{m}$, the $\sigma_{11}$ value computed at the $3^{\text {rd }}$ and deeper nodes are systematically superimposed on the exact analytical stress profile (Fig $4 b$ ). This suggests that to complete a relevant fatigue stress analysis, the analysis must consider the stress field at $\mathrm{n} \geq 3$ nodal position to the surface to avoid any mesh size influence on the prediction. It is interesting to note that the necessity to consider a subsurface stress description is compatible with a non-local critical distance strategy.

Indeed, as developed by many authors, when severe stress gradients are imposed like fretting or notch configurations, the fatigue analysis cannot be performed at the hot spot stress because it induces critical overestimation of the cracking risk [6]. Non local fatigue stress field description 
must be preferred [7]. One strategy extensively developed by Taylor's and Susmel [8] and transposed by Araújo for fretting problems [9] consists in considering the stress field at a critical distance $\ell$ deeper from the hot spot.

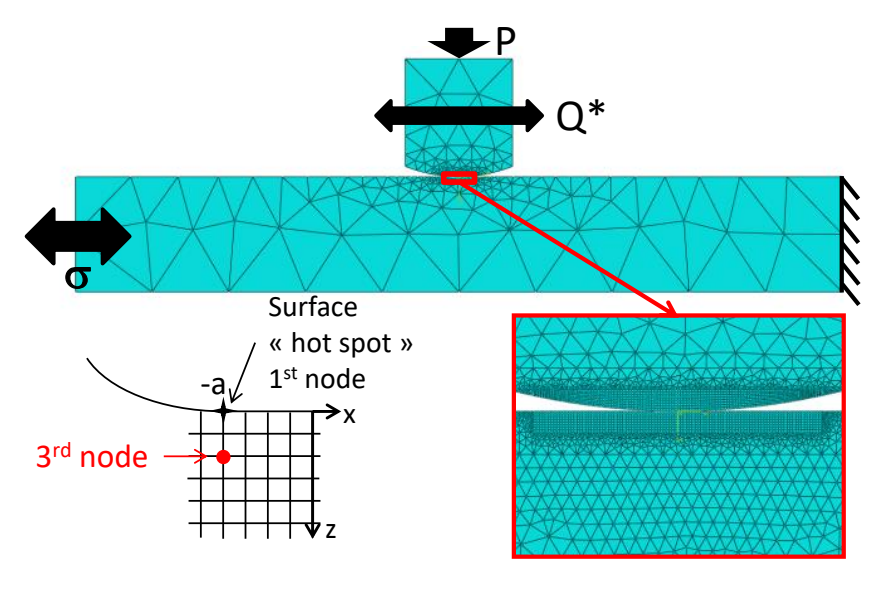

(a)

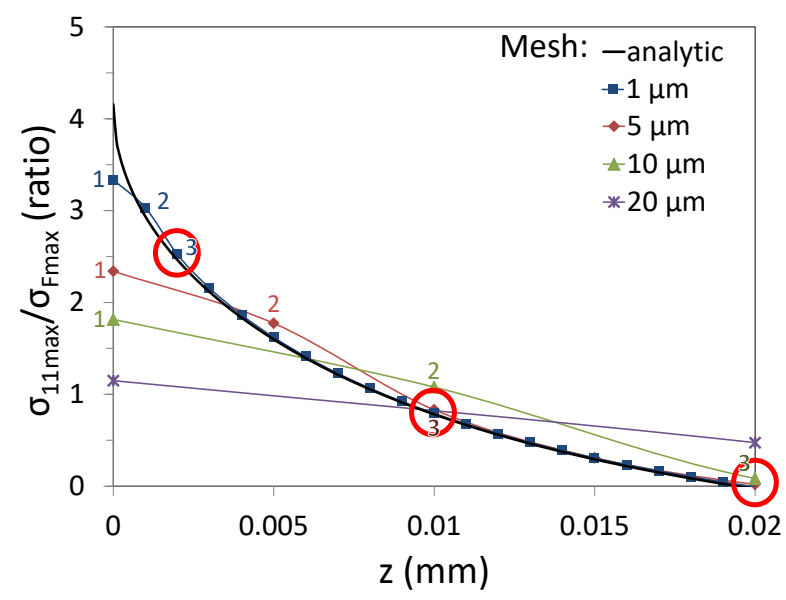

(b)

Fig. 4. (a) Abaqus FEM model of the fretting fatigue test (b) Elastic $\sigma_{11}(x=-a, z)$ profiles at the trailing contact border for a given plain fretting condition $(\mathrm{P}, \mathrm{Q} / \mu \mathrm{P}=0.22)$

A major question is to extrapolate a relevant value of $\ell$. Former analysis [2] shows that this value can, for a given $b_{p_{-} x}$ crack nucleation threshold, be extrapolated from a reverse analysis of plain fretting cracking experiments by solving the following expression:

$$
\sigma_{\mathrm{eq}}\left(\ell_{\mathrm{x}}\right)=\tau_{\mathrm{d}}
$$

with $\sigma_{e q}$ an equivalent fatigue stress defined below the surface et $\left(z=\ell_{x}\right)$ and $\tau_{d}$ the alternated shear fatigue limit. Considering that the fretting fatigue stress field below the surface is multi-axial, the Crossland [10] approach is considered to establish the so-called Crossland equivalent stress:

$$
\sigma_{C}=\sqrt{J_{2, a}}+\alpha \sigma_{H, \max } \text {. }
$$

Where $\alpha=\left(\tau_{d}-\frac{\sigma_{d}}{\sqrt{3}}\right) /\left(\frac{\sigma_{d}}{\sqrt{3}}\right)$

with $\sigma_{\mathrm{d}}$ the alternated bending fatigue limit, $\sqrt{\mathrm{J}_{2, \mathrm{a}}}$ the maximum amplitude of the second invariant of the stress deviator and $\sigma_{\mathrm{H}, \max }$ the maximum value of the hydrostatic pressure. For $\sigma_{C} \geq \tau_{d}$ there is a cracking risk. To be consistent with the FEM mesh size effect observed, a mesh size condition is introduced:

$$
\mathrm{d}_{\mathrm{X}} \leq \ell_{\mathrm{X}} / 2
$$

with $d_{x}$ the fretting surface mesh size related to the $b_{p_{-} x}$ crack analysis threshold. In this reverse analysis, the contact mesh size was fixed at $d=1 \mu \mathrm{m}$. Hence, the mesh size condition is systematically satisfied. A reverse analysis satisfying Eq. 1 is therefore adapted to establish, from the plain fretting nucleation conditions, the critical distance $\ell_{\mathrm{x}}$ related to each crack nucleation length condition (Table 3 ).

Table 3: Extrapolated critical distance values $\boldsymbol{\ell}_{\mathrm{x}}$ for $\mathrm{b}_{\mathrm{x}_{-} \mathrm{th}}=0,50$ and $100 \mu \mathrm{m}$

\begin{tabular}{l|c|c|c}
$b_{p_{-} t h}[\mu \mathrm{m}]$ & 0 & 50 & 100 \\
\hline$Q^{*} / \mu . P[\mathrm{~N} / \mathrm{mm}]$ & 0.13 & 0.23 & 0.30 \\
\hline$\ell_{x}[\mu \mathrm{m}]$ & 15 & 30 & 40 \\
\hline
\end{tabular}

Formalization. Using the $\ell_{\mathrm{x}}$ values extrapolated from the plain fretting analysis, the various experimental fretting fatigue crack nucleation conditions are compared versus the material 
boundary in the so called $\sqrt{J_{2, a}}-\sigma_{H, \max }$ diagram. The material boundary was expressed for $10^{6}$ cycles through the corresponding fatigue limit $\sigma_{\mathrm{d}-1}$ and $\sigma_{\mathrm{d} 0.1}$ at $10^{6}$ cycles. Note that this FEM fatigue analysis was performed considering the elastic-plastic response of the material and using a $\mathrm{d}=5 \mu \mathrm{m}$ contact mesh size (i.e. $\left.\mathrm{d} \leq \ell_{\mathrm{x}} / 2\right)$.

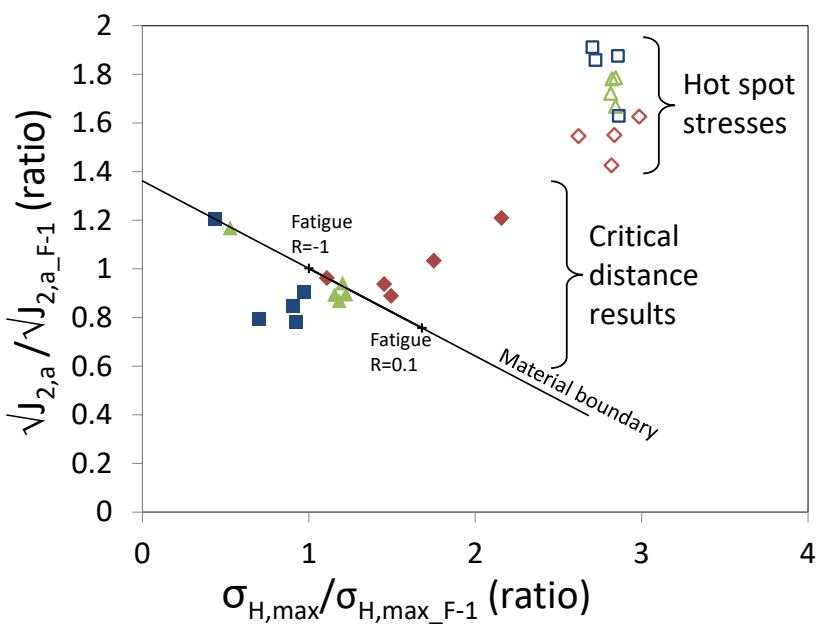

(a)

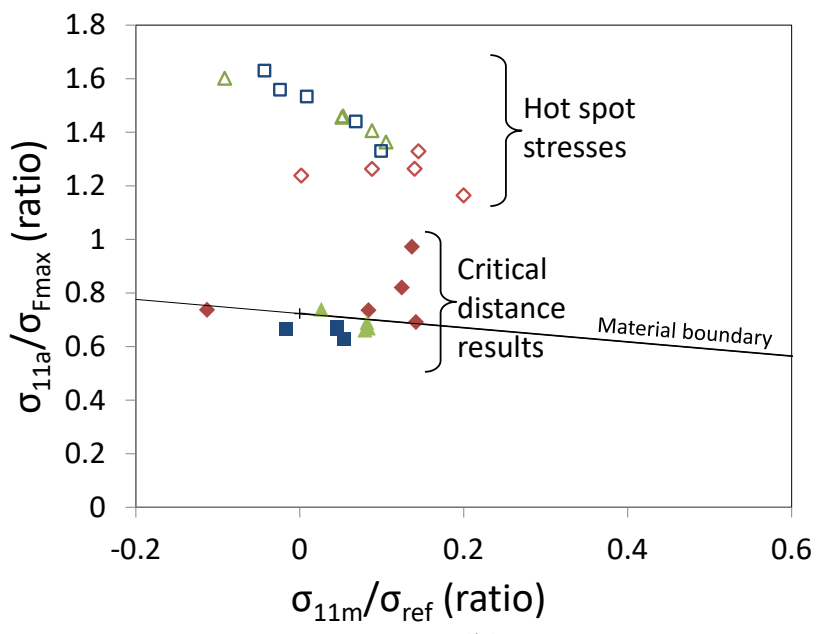

(b)

Fig. 5. Fatigue analysis of fretting fatigue crack nucleation experiments $\left(\mathrm{P} ; \sigma_{\mathrm{Fmax}} ; \mathrm{R}_{\mathrm{F}}=0.6\right.$ to 1 ; EP laws; $\mathrm{d}=5 \mu \mathrm{m} ; \mu=1)$.

Hot spot analysis $(\mathrm{z}=\ell=0 \mu \mathrm{m}): \square \mathrm{b}_{\mathrm{p}_{-} \mathrm{th}}=100 \mu \mathrm{m} ; \Delta \mathrm{b}_{\mathrm{p}_{-} \mathrm{th}}=50 \mu \mathrm{m} ; \diamond \mathrm{b}_{\mathrm{p}_{-} \mathrm{th}}=0 \mu \mathrm{m}$

Critical distance analysis $\left(\mathrm{z}=\ell_{\mathrm{x}}>0 \mu \mathrm{m}\right): \Delta \mathrm{b}_{\mathrm{p}_{-} \mathrm{th}}=100 \mu \mathrm{m} ; \Delta \mathrm{b}_{\mathrm{p}_{-} \mathrm{th}}=50 \mu \mathrm{m} ; \bullet \mathrm{b}_{\mathrm{p}_{-} \mathrm{th}}=0 \mu \mathrm{m}$

(a) $\sqrt{J_{2, a}}-\sigma_{H, \max }$ representation: comparison between hot spot stresses $(\mathrm{z}=0, \mathrm{x}=-\mathrm{a})$ and critical distance analyses $\left(\mathrm{z}=\ell_{\mathrm{x}}, \mathrm{x}=-\mathrm{a}\right)$ (Table 3$)$.

(b) Equivalent $\sigma_{11 \mathrm{a}}-\sigma_{11 \mathrm{~m}}$ uni-axial Haigh diagram: comparison between hot spot stresses ( $\mathrm{z}=0$, $\mathrm{x}=-\mathrm{a})$ and critical distance analyses $\left(\mathrm{z}=\ell_{\mathrm{x}}, \mathrm{x}=-\mathrm{a}\right)$ (Table 3$)$.

As expected from [4], in addition to not considering reliable stress estimation, the surface hot spot fatigue stress analysis overestimates the cracking risk (Fig. 5a). By contrast he results provided by the critical distance approach are more consistent. The crack nucleation conditions are gathered along the material boundary. However, if the $b_{p_{-} t h}=50 \mu \mathrm{m}$ results are really close to the material boundary whatever the $\mathrm{R}_{\mathrm{F}}$ ratio, the predictions provided for the longest crack length $\mathrm{b}_{\mathrm{p}_{-} \mathrm{th}}=100 \mu \mathrm{m}$ appear non-conservative (i.e. below the theoretical boundary), whereas the predictions related to the $\mathrm{b}_{\mathrm{p}_{-} \mathrm{t}}=0 \mu \mathrm{m}$ are too conservative. This suggests that the crack nucleation must be calibrated regarding a representative incipient crack length. If the chosen crack length is too small (i.e. $b_{p_{-} t h}=0 \mu m$ ) the studied crack length is probably not representative of a damaging crack according that such very small crack may never propagate and therefere is not representative of the conventional fatigue limit. By contrast, if the chosen crack length is too long, it is probably also controlled by a crack propagation process rather than only a crack nucleation phenomenon. This analysis suggests that a reliable crack nucleation analysis is achieved choosing a threshold crack length $\mathrm{b}_{\mathrm{p}_{-} \mathrm{th}}=50 \mu \mathrm{m}$.

As previously developed in [2-4], the fretting fatigue stress field is nearly uni-axial at least on the top surface hot spot vicinity. Therefore, the crack nucleation process could be described using simpler Haigh's uni-axial approach, where the crack nucleation conditions are reported as a function of the total $\sigma_{11}$ mean $\left(\sigma_{11 \mathrm{~m}}\right)$ and amplitude $\left(\sigma_{11 \mathrm{a}}\right)$ stress component [3]. Because both fretting and fatigue loadings are in phase, both fatigue and fretting components can be added so that:

$$
\begin{aligned}
& \sigma_{11 \mathrm{a}}=\sigma_{11 \mathrm{a} \text { (fatigue) }}+\sigma_{11 \mathrm{a} \text { (fretting). }} \\
& \sigma_{11 \mathrm{~m}}=\sigma_{11 \mathrm{~m} \text { (fatigue) }}+\sigma_{11 \mathrm{~m} \text { (fretting). }}
\end{aligned}
$$

Fig $5 \mathrm{~b}$ compares the results achieved using hot spot and critical distance methods (Table 3 ). Similar conclusion can be driven. The best correlation is achieved using the critical distance 
approach. The results obtained for $b_{p_{-} t h}=50 \mu \mathrm{m}$ and $b_{p_{-} t h}=100 \mu \mathrm{m}$ are very close to the material boundary whereas the crack nucleation conditions related to $b_{\mathrm{p}_{\_} \mathrm{th}}=0 \mu \mathrm{m}$ are slightly over estimated. This suggests that by using a very basic uni-axial fatigue description, reliable crack nucleation predictions of fretting fatigue contacts can be achieved if pertinent critical distance approach is applied in relation with adequate contact FEM meshing $\left(\mathrm{d} \leq \ell_{\mathrm{x}} / 2\right)$.

\section{Conclusion}

This analysis focuses on the effect of fatigue stress ratio $R_{F}$ regarding the fretting fatigue crack nucleation condition. The following aspects have been underlined:

- At constant maximal fatigue force, the effect of $\mathrm{R}_{\mathrm{F}}$ depends on the threshold crack length defining the crack nucleation condition. If $b_{p_{-} t h}=0 \mu \mathrm{m}$, the cracking process is only controlled by the cyclic tangential force amplitude. For larger $b_{\mathrm{p}_{-} \text {th }}$, the threshold $\mathrm{Q}^{*}$ tangential force is reduced with increasing $R_{F}$.

- The FEM analysis required to compute the elastic-plastic stress field is mesh size dependent. A reliable stress field description is achieved only below the $3^{\text {rd }}$ node along the $\mathrm{z}$ axis at the trailing contact border (i.e., $2^{\text {nd }}$ node below the surface).

- Reliable predictions of fretting fatigue cracking risk are achieved using a critical distance approach $\left(\ell_{\mathrm{x}}\right)$ where the critical distance value related to a given threshold crack length $\left(\mathrm{X}=\mathrm{b}_{\mathrm{p}_{-} \mathrm{th}}\right)$ is defined from plain fretting experiment. This good prediction implies to consider an elastic-plastic stress field analysis, a node size condition $\left(\mathrm{d} \leq \ell_{\mathrm{x}} / 2\right)$ and a representative crack nucleation length $\mathrm{b}_{\mathrm{p}_{-} \mathrm{th}}=50 \mu \mathrm{m}$.

- Due to a quasi uni-axial stress condition at the trailing contact vicinity, the fretting fatigue crack nucleation condition can be addressed using a very basic $\sigma_{11 \mathrm{a}}-\sigma_{11 \mathrm{~m}}$ Haigh description.

\section{References}

[1] R.B. Waterhouse, Fretting Fatigue, Waterhouse, 1981.

[2] S. Fouvry, H. Gallien, B. Berthel, From uni- to multi-axial fretting-fatigue crack nucleation: Development of a stress-gradient-dependent critical distance approach, Int. J. Fatigue. (2013).

[3] S. Fouvry, F. Ruiz, P. Kapsa, L. Vincent, Stress and Fatigue analysis of fretting on a stressed specimen under partial slip condition, Tribotest J. 3 (1996) 1354-4063.

[4] R. Ferré, S. Fouvry, B. Berthel, J. -a. Ruiz-Sabariego, Stress gradient effect on the crack nucleation process of a $\mathrm{Ti}-6 \mathrm{Al}-4 \mathrm{~V}$ titanium alloy under fretting loading: Comparison between non-local fatigue approaches, Int. J. Fatigue. 54 (2013) 56-67.

[5] S. Heredia, S. Fouvry, B. Berthel, E. Greco, Introduction of a "principal stress-weight function" approach to predict the crack nucleation risk under fretting fatigue using FEM modeling, Submitt. to IJF. (n.d.).

[6] J. Araújo, D. Nowell, The effect of rapidly varying contact stress fields on fretting fatigue, 24 (2002) $763-775$.

[7] S. Fouvry, P. Kapsa, F. Sidoroff, L. Vincent, Identification of the characteristic length scale for fatigue cracking in fretting contacts, J Phys IV Fr. 8 (1998) 66.

[8] L. Susmel, D. Taylor, Can the conventional High-cycle multiaxial fatigue criteria be re-interpreted in terms of the theory of critical distances?, SDHM. 2 (2006) 91-108.

[9] J. Araújo, L. Susmel, D. Taylor, J.C.T. Ferro, J.L. a. Ferreira, On the prediction of high-cycle fretting fatigue strength: Theory of critical distances vs. hot-spot approach, Eng. Fract. Mech. 75 (2008) 1763-1778.

[10] B. Crossland, Effect of large hydrostatic pressures on the torsional fatigue strength of an alloy steel, Proc. Int. Conf. Fatigue Met. (1956) 138-149. 